\title{
BACTERIEMIA POR LISTERIA EN PACIENTES DE UN INSTITUTO ONCOLÓGICO PERUANO, 2005-2015
}

\author{
Luis E. Cuéllar(11,2,a, Alfredo Chiappe (103,a, Juan Velarde (101,2,a, Alexis Holguín(101,a, \\ Diana Portillo@1,a, William Vicente(10,b \\ ${ }^{1}$ Servicio de Infectología, Instituto Nacional de Enfermedades Neoplásicas, Lima, Perú. \\ ${ }^{2}$ Universidad Nacional Federico Villarreal, Lima, Perú. \\ ${ }^{3}$ Servicio de Enfermedades Infecciosas y Tropicales, Hospital Nacional Dos de Mayo, Lima, Perú. \\ ${ }^{4}$ Servicio de Microbiología, Instituto Nacional de Enfermedades Neoplásicas, Lima, Perú. \\ ${ }^{a}$ Médico infectólogo; ${ }^{\mathrm{b}}$ médico patólogo clínico.
}

\section{RESUMEN}

La listeriosis es una infección severa de elevada morbimortalidad en el paciente inmunocomprometido, existen formas diseminadas y fatales en el paciente oncológico. Se realizó un estudio descriptivo de las características clínico-epidemiológicas de los pacientes oncológicos con listeriosis atendidos en el Instituto Nacional de Enfermedades Neoplásicas entre 2005 y 2015. Se incluyeron 29 pacientes, 23 (79,3\%) casos de listeriosis se presentaron en pacientes con neoplasia hematológica, de los cuales el 52,3\% fueron leucemia linfática aguda y $39,1 \%$ linfoma no Hodgkin. Listeria monocytogenes representó el 72,4\% de las especies aisladas. Veintisiete $(93,1 \%)$ tuvieron criterios de sepsis y veinticuatro $(82,7 \%)$ compromiso neurológico al ingreso. La presentación más común fue la bacteriemia, seguida de la meningoencefalitis (20,6\%). La mortalidad global fue del $75,8 \%$. Se concluye que la listeriosis conlleva elevada morbimortalidad en el paciente oncohematológico, por lo que debe sospecharse en el paciente admitido con sepsis y/o compromiso neurológico agudo.

Palabras clave: Listeria; Neoplasias; Bacteriemia; Perú (fuente: DeCS BIREME).

\section{LISTERIA BACTEREMIA IN PATIENTS FROM A PERUVIAN ONCOLOGIC INSTITUTE, 2005-2015}

\begin{abstract}
Listeriosis infection is a severe disease, with high morbidity and mortality in the immunocompromised patient, especially with disseminated and fatal presentations in cancer patients. A descriptive study was developed to describe the clinical and epidemiologic characteristics in oncologic patients with listeriosis in the Instituto Nacional de Enfermedades Neoplásicas between the years 2005-2015. A total of 29 patients were included; $23(79.3 \%)$ of the listeriosis cases showed up in patients with hematological neoplasia, of which $52.1 \%$ was acute lymphatic leukemia and $39.1 \%$ non-Hodgkin's lymphoma. The $72.4 \%$ of the isolated species correspond to Listeria monocytogenes. Twenty-seven $(93.1 \%)$ patients met sepsis criteria and twenty-four $(82.7 \%)$ had neurologic affection. Bacteremia was the most common presentation, followed by meningoencephalitis (20.6\%). Global mortality was $75.8 \%$. In conclusion in cancer patients, listeriosis implies high morbidity and mortality. Therefore, the suspicion of this entity is mandatory in onco-hematologic patients with sepsis and acute neurologic symptoms.
\end{abstract}

Citar como: Cuéllar LE, Chiappe A, Velarde J, Holguín A, Portillo D, Vicente W. Bacteriemia por listeria en pacientes de un instituto oncológico en peruano, 2005-2015. Rev Peru Med Exp Salud Publica. 2021;38(1):10812. doi: https://doi.org/10.17843/ rpmesp.2021.381.5488.

Correspondencia: Alfredo Juan Chiappe Gonzalez; Av. Del Sur 237, Dpto 203, Santiago de Surco, Lima, Perú; alfredochiappe911@hotmail.com

Recibido: 04/04/2020 Aprobado: 04/11/2020 En línea: 04/03/2021
Keywords: Listeria; Neoplasms; Bacteremia; Peru (source: MeSH NLM).

\section{INTRODUCCIÓN}

Listeria monocytogenes es un bacilo grampositivo, anaerobio facultativo, responsable del $90 \%$ de las infecciones humanas por el género Listeria ${ }^{(1)}$. La listeriosis se manifiesta desde gastroenteritis autolimitadas en el inmunocompetente, hasta bacteriemias, meningoencefalitis y rombencefalitis, producto de la diseminación y mayor severidad en el inmunocomprometido. En gestantes puede ocasionar aborto u óbito fetal ${ }^{(1,2)}$.

En más del 70\% de casos, la listeriosis se presenta en grupos vulnerables como son los pacientes inmunocomprometidos, mayores de 60 años, neonatos, gestantes, hepatopatía, diabetes y cáncer; representando esta última más del $30 \%$ del total de los casos ${ }^{(3)}$. En pacientes con cáncer, la listeriosis se observa con mayor frecuencia en la leucemia linfocítica crónica 
(21\%), leucemia linfocítica aguda (LLA) (12\%) y el linfoma Hodgkin (12\%), con una mortalidad de hasta $32 \%$ comparada con el $27 \%$ en otros pacientes vulnerables ${ }^{(4,5)}$.

En el paciente con cáncer, la mayor frecuencia y severidad de listeriosis radica en el mayor número de factores de riesgo ${ }^{(5)}$; sin embargo, aún es escasa la información disponible en la literatura médica sobre este tema. En tal sentido, se presenta un estudio descriptivo acerca de las características clínicas y epidemiológicas de los pacientes con listeriosis atendidos en el Instituto Nacional de Enfermedades Neoplásicas entre 2005 y 2015.

\section{EL ESTUDIO}

Se identificaron 29 casos de listeriosis a través de la revisión de la base de datos de cultivos del Departamento de Microbiología del Instituto Nacional de Enfermedades Neoplásicas (INEN) en el periodo 2005-2015. La información de los pacientes referente a aspectos epidemiológicos, comorbilidades, neoplasia, manifestaciones clínicas, laboratorio e imágenes, así como el tratamiento antibiótico instaurado, corticoterapia, agentes quimioterápicos y desenlace clínico, se obtuvo de las historias clínicas.

Se consideró como paciente adulto mayor a aquel con 65 años o más. El diagnóstico de listeriosis se estableció si el paciente tenía signos o síntomas de infección, asociado a un cultivo positivo de sangre y/o de líquido cefalorraquídeo (LCR) para especies de Listeria. La meningoencefalitis confirmada por Listeria se definió como aquel paciente con compromiso del sistema nervioso central (SNC), ya sea con trastorno del sensorio, convulsiones y/o signos meníngeos, asociado a fiebre, pleocitosis, con o sin bacteriemia por Listeria, con o sin alteraciones en los estudios de imágenes y cultivo de LCR positivo. Se estableció el diagnóstico de sepsis por Listeria a la presencia del síndrome de respuesta inflamatoria sistémica, asociado al cultivo positivo. Se definió neutropenia como una recuento de neutrófilos $<1000$ por $\mathrm{mL}$ y linfopenia como $<1000$ linfocitos/mL. En los estudios de LCR se consideró proteinorraquia como aquella $>600$ $\mathrm{mg} / \mathrm{L}$, pleocitosis $>5$ células $/ \mathrm{mL}$ y consumo de glucosa al resultado del índice glucosa LCR/glucosa sérica $<0,4$.

Las pruebas de sensibilidad antibiótica se realizaron mediante la técnica de difusión en disco (Kirby-Bauer), de acuerdo con estándares internacionales del Clinical and Laboratory Standards Institute (CLSI). La concentración inhibitoria mínima (CIM) para algunos antibióticos se obtuvo de los resultados emitidos por el sistema automatizado BD Phoenix, aunque no existen puntos de corte para este microorganismo. En algunas ocasiones se emitieron la CIM sin puntos de corte de referencia o en su defecto se tomaron en cuenta los puntos de corte de European Committee on Antimicrobial Susceptibility Testing (EUCAST).

El diagnóstico que motivó el inicio antibiótico fue la neutropenia febril. La terapia empírica consistió en merope-

\section{MENSAJES CLAVE}

Motivación para realizar el estudio: En el paciente con cáncer, la listeriosis tiene un comportamiento diseminado con desenlaces frecuentemente fatales. La terapia empírica suele ser tardía por falta de sospecha clínica.

Principales hallazgos: El 79,3\% de casos de listeriosis se presentaron en pacientes con neoplasia hematológica. Al ingreso, veintisiete $(93,1 \%)$ pacientes cumplían criterios de sepsis y veinticuatro $(82,7 \%)$ tenían algún tipo de compromiso neurológico: trastorno del sensorio $(79,3 \%)$, signos meníngeos $(51,7 \%)$ y convulsiones $(24,1 \%)$.

Implicancias: Debe sospecharse de listeriosis en todo paciente oncohematológico que curse con sepsis y compromiso neurológico agudo hasta la obtención de cultivos.

nem en ocasiones asociado a vancomicina, a lo que se agregó ampicilina (12 g/día) una vez obtenido el resultado de bacilos grampositivos en sangre o LCR. La respuesta a la terapia antibacteriana se definió como la resolución de los signos o síntomas de la infección, incluida la negativización de los cultivos o la mejoría imagenológica. Por otro lado, la falla a la terapia antibacteriana se definió como el deterioro clínico del paciente, incluyendo empeoramiento de los parámetros laboratoriales e imagenológicos con desenlace fatal.

El desenlace clínico se evaluó por el seguimiento del paciente registrado en la historia clínica hasta los 60 días posteriores al cultivo positivo para Listeria, catalogándose como respuesta o falla al tratamiento antibacteriano.

Se realizó un análisis descriptivo mediante el programa estadístico Stata 12.0 (Stata Corporation, College Station, Texas, EUA), con el cual se obtuvieron frecuencias y porcentajes para las variables cualitativas y media con su desviación estándar o mediana con su rango intercuartil (RIQ) para las de naturaleza cuantitativa.

La investigación contó con aprobación del Comité Revisor de Protocolos del Departamento de Investigación del INEN previo a su realización (INEN $N^{\circ} 16-57$ ). No se desarrolló ningún tipo de intervención sobre seres humanos. Se mantuvo el derecho de confidencialidad de los registros clínicos analizados.

\section{HALLAZGOS}

El $65,5 \%$ de casos registrados en la última década se concentraron a partir del 2010 hasta el 2015, con porcentajes menores durante los años previos. Se obtuvo una mayor distribución durante los meses de verano y otoño (62\%); con cifras menores durante el invierno (17\%). 
Se identificaron 29 pacientes oncológicos con listeriosis con mediana de edad de 54 años (RIQ 11-61). Veintitrés casos $(79,3 \%)$ se presentaron en neoplasia hematológica y 20,6\% neoplasia sólida (Tabla 1). Adicionalmente a la neoplasia de fondo, hubieron 9 (31\%) pacientes con otras comorbilidades: hipertensión arterial $(\mathrm{n}=2)$ y cirrosis hepáti$\mathrm{ca}$, diabetes mellitus, hipotiroidismo, esquizofrenia, déficit G6PD, infección VHB e infección HTLV 1-2 con un caso por patología.

En 18 (62\%) pacientes hubo uso previo de bloqueadores anti-H2 o inhibidores de bomba de protones (IBP). Veintisiete $(93,1 \%)$ se hallaban recibiendo corticoides al momento de la infección. Los esquemas quimioterápicos se detallan en la Tabla 2.

Tabla 1. Características de pacientes con listeriosis en el Instituto $\mathrm{Na}-$ cional Enfermedades Neoplásicas, 2005-2015 ( $\mathrm{n}=29$ ).

\begin{tabular}{|c|c|}
\hline Características & n (\%) \\
\hline \multicolumn{2}{|l|}{ Edad (años) } \\
\hline$<18$ & $9(31,0)$ \\
\hline$<18-65$ & $14(48,3)$ \\
\hline$>65$ & $6(20,7)$ \\
\hline \multicolumn{2}{|l|}{ Sexo } \\
\hline Masculino & $17(58,6)$ \\
\hline Femenino & $12(41,4)$ \\
\hline \multicolumn{2}{|l|}{ Neoplasia de fondo } \\
\hline Neoplasia hematológica & $23(79,3)$ \\
\hline Tumor sólido & $6(20,7)$ \\
\hline \multicolumn{2}{|l|}{ Neoplasia hematológica } \\
\hline Leucemia linfática aguda & $12(52,3)$ \\
\hline Leucemia mieloide aguda & $1(4,3)$ \\
\hline Linfoma no Hodgkin & $9(39,1)$ \\
\hline Mieloma múltiple & $1(4,3)$ \\
\hline \multicolumn{2}{|l|}{ Tumor sólido } \\
\hline Neoplasia pulmón & $2(33,2)$ \\
\hline Neoplasia mama & $1(16,7)$ \\
\hline Neoplasia cérvix & $1(16,7)$ \\
\hline Sarcoma sinovial & $1(16,7)$ \\
\hline Enfermedad trofoblástica gestacional & $1(16,7)$ \\
\hline \multicolumn{2}{|l|}{ Tiempo de hospitalización (días) } \\
\hline$<5$ & $11(37,9)$ \\
\hline 5 o más & $18(62,1)$ \\
\hline \multicolumn{2}{|l|}{ Presentación } \\
\hline Intrahospitalario & $10(34,5)$ \\
\hline Extrahospitalario & $19(65,5)$ \\
\hline
\end{tabular}

Presentación intrahospitalaria: listeriosis desarrollada durante internamiento. Presentación extrahospitalaria: listeriosis como parte del cuadro clínico de ingreso.
Tabla 2. Esquemas quimioterápicos en pacientes con listeriosis del Instituto Nacional Enfermedades Neoplásicas, 2005-2015 ( $\mathrm{n}=29$ ).

\begin{tabular}{lc}
\hline Esquema quimioterápico & $\mathbf{n}(\%)$ \\
\hline Vincristina-DNM-L asparaginasa & $7(24,1)$ \\
CHOP & $5(17,2)$ \\
Paclitaxel-Carboplatino & $2(6,9)$ \\
Alemtezumab & $1(3,4)$ \\
Otros & $14(48,3)$ \\
\hline
\end{tabular}

DNM: daunorrubicina; CHOP: esquema quimioterápico constituido por vincristina, doxorrubicina, ciclofosfamida y prednisona.

Al ingreso, veintisiete $(93,1 \%)$ cumplían criterios de sepsis y veinticuatro $(82,7 \%)$ tenían algún tipo de compromiso neurológico [trastorno del sensorio: 23 (79,3\%), signos meníngeos: 15 (51,7\%) y convulsiones: $7(24,1 \%)]$. La presentación más común fue bacteriemia, observada en los veintinueve pacientes, en seis de los cuales (20,6\%) hubo también cultivo positivo en el LCR. La superinfección asociada más frecuente fue bacteriemia por Pseudomonas aeruginosa $(10,3 \%)$.

Se reportaron aislamientos de Listeria sp. durante periodos de tiempo determinados, debido a limitaciones en laboratorio para identificar la especie implicada. Listeria monocytogenes representó el $72,4 \%$ de las especies aisladas (Tabla 3). Ampicilina, vancomicina y meropenem fueron los antibióticos empíricamente más empleados. Se identificó sensibilidad in vitro a ampicilina en 21 (84\%) pacientes (Tabla 4). Cuatro pacientes presentaron secuelas neurológicas $(13,7 \%)$, de los cuales el 50\% tenían cultivo en LCR positivo. De los 29 pacientes con bacteriemia, 75,8\% fallecieron (mortalidad global); mientras que la mortalidad global en meningoencefalitis confirmada fue $16,6 \%$. No fue posible calcular la mortalidad atribuible a listeriosis.

\section{DISCUSIÓN}

La listeriosis es un problema de salud pública, por ser una de las enfermedades trasmitidas por alimentos más frecuentes. En los Estados Unidos, a pesar de presentar una incidencia baja en la población general (0,1 a 1 casos por 100000 habitantes), es responsable del 19\% de las muertes por ingesta de alimentos contaminados de etiología conocida, cifra similar a la francesa, donde representa $17 \%$ del total ${ }^{(6,7)}$. Un estudio transversal realizado en la ciudad de Ica (Perú), recolectó 74 muestras de queso fresco de leche de vaca de producción artesanal, procedente de los principales mercados abastecedores al público de la localidad. Se halló 28 aislamientos $(20,7 \%)$ del total de muestra, contaminada por colonias sugerentes de listeria, la leche de vaca fue la fuente probable de contaminación durante la elaboración ${ }^{(8)}$. 
Tabla 3. Características laboratoriales, imagenológicas y microbiológicas de pacientes con listeriosis en el Instituto Nacional Enfermedades Neoplásicas, 2005-2015 ( $n=29)$.

\begin{tabular}{|c|c|}
\hline Características & n (\%) \\
\hline \multicolumn{2}{|l|}{ Leucocitos (cel/mL) } \\
\hline$<4000$ & $14(48,3)$ \\
\hline $4000-12000$ & $11(37,9)$ \\
\hline$>12000$ & $4(13,8)$ \\
\hline \multicolumn{2}{|l|}{ Neutrófilos (cel/mL) } \\
\hline$<100$ & $8(27,6)$ \\
\hline $100-500$ & 0 \\
\hline $500-1000$ & $1(3,4)$ \\
\hline$>1000$ & $20(69,0)$ \\
\hline \multicolumn{2}{|l|}{ Linfocitos (cel/mL) } \\
\hline$<1000$ & $27(93,1)$ \\
\hline \multicolumn{2}{|l|}{ LCR } \\
\hline Proteínas $>600 \mathrm{mg} / \mathrm{L}$ & $17(85,0)$ \\
\hline$>5$ células & $16(80,0)$ \\
\hline Id. glucosa LCR/ glucosa sérica $<0,4$ & $9(45,0)$ \\
\hline \multicolumn{2}{|l|}{ TAC cerebro } \\
\hline Absceso cerebral/cerebritis & $2(9,5)$ \\
\hline Signos indirectos de meningitis & $1(4,8)$ \\
\hline Infiltración secundaria & $4(19,0)$ \\
\hline Otros & $6(28,6)$ \\
\hline Sin alteraciones significativas & $8(38,1)$ \\
\hline \multicolumn{2}{|l|}{ Especie microbiológica } \\
\hline Listeria monocytogenes & $21(72,4)$ \\
\hline Listeria ivanovii & $1(3,4)$ \\
\hline Listeria sp. & $7(24,1)$ \\
\hline
\end{tabular}

LCR: líquido cefalorraquídeo; TAC: tomografía axial computarizada; Id: índice.

Los resultados del estudio muestran mayor concentración de casos en los últimos cinco años, con mayor frecuencia durante los meses de verano y otoño, lo cual se halla acorde a otros reportes, donde los meses estivales, sobre todo en países del hemisferio norte presentan un mayor número de casos ${ }^{(0,10)}$. Cairns et al., asocian matemáticamente el incremento en el número de casos con el aumento de la temperatura climática ${ }^{(10)}$. Sin embargo, los resultados descritos difieren con los de Sedano et al., quienes reportan en Chile que el 10\% de casos ocurren en los meses de verano, justificando ello al mayor consumo de verduras congeladas contaminadas en los meses restantes, las cuales servirían como vehículo de transmisión ${ }^{(11)}$.
Tabla 4. Perfil de sensibilidad antibiótica.

\begin{tabular}{lc}
\hline Sensibilidad antibiótica & $\mathbf{n}(\%)$ \\
\hline Ampicilina & $21(84)$ \\
Gentamicina & $24(85,7)$ \\
Cotrimoxazol & $17(77,2)$ \\
Penicilina & $3(75,0)$ \\
Meropenem & $27(100)$ \\
Vancomicina & $25(96,1)$ \\
\hline
\end{tabular}

La listeriosis en el paciente oncológico se manifiesta frecuentemente en aquellos con neoplasia hematológica, y se presentó un mayor número en los casos de leucemia linfática aguda y en linfoma no Hodgkin (LNH), ambas neoplasias linforeticulares. El control de la infección por Listeria monocytogenes requiere de la inmunidad celular al tratarse de un agente intracelular, necesitando una adecuada respuesta tipo T helper 1, con participación concomitante de macrófagos por parte de la inmunidad innata ${ }^{(12,13)}$. La LLA y el LNH, que representan más del 70\% de casos de la serie descrita, se caracterizan por ser neoplasias que alteran la inmunidad celular, predisponiendo a enfermedad con formas graves y diseminadas. Se suman como factores agravantes, la edad avanzada, corticoterapia y linfopenia, observados en $20 \%$, $93 \%$ y $93 \%$ de casos respectivamente.

Al tratarse de una infección cuya puerta de ingreso es el tracto gastrointestinal, una de las primeras líneas defensivas es la acidez del jugo gástrico, por lo cual se ha descrito que el consumo de medicamentos que modifiquen el $\mathrm{pH}$ gástrico (alcalinizándolo), podrían ser factores de riesgo para desarrollo y progresión de enfermedad ${ }^{(14)}$. El uso de IBP o antagonistas $\mathrm{H} 2$ en la serie descrita fue del $62 \%$ del total de casos, lo que podría haber jugado como factor de riesgo adicional.

Concordante a la literatura, la bacteriemia a manera de sepsis fue la manifestación más frecuente $(93,1 \%)$, seguida de meningoencefalitis confirmada $(20,6 \%)^{(12-14)}$. No obstante, se observó que el 82,7\% de pacientes presentó algún tipo de compromiso neurológico agudo al ingreso, se plantea que posiblemente existan subdiagnósticos de meningoencefalitis por Listeria sp.

En la serie de casos evaluada, $34,5 \%$ de casos ocurrió durante la hospitalización, cifra bastante elevada al contrastarla con otras series que oscilan entre el 8,7\% al 30\% de los casos ${ }^{(15)}$. Los brotes nosocomiales se han asociado al consumo de alimentos contaminados, por lo que es importante reforzar el estricto control de calidad en la administración de alimentos en las instituciones proveedoras de salud para pacientes oncohematológicos. Otra hipótesis plantea el estado de portador asintomático, cuya prevalencia es del $10 \%$ según reportes, con posterior expresión clínica de enfermedad luego de terapia inmunosupresora ${ }^{(16)}$.

Interesante resulta el aislamiento en hemocultivo de Listeria ivanovii, la cual ha sido descrita como patógeno única- 
mente en rumiantes. Existen reportes extremadamente raros de enfermedad en humanos severamente inmunocomprometidos. Se describe gastroenteritis y bacteriemia, siendo considerado como posible patógeno humano entérico oportunista ${ }^{(17)}$.

El diagnóstico y tratamiento de listeriosis debe ser oportuno y precoz. Meropenem con/sin vancomicina fue la terapia empírica más empleada, debido a que la neutropenia febril fue el diagnóstico inicial. Luego, ampicilina fue agregada al reportarse bacilos gran positivos en las muestras de sangre o LCR. Ampicilina asociada a gentamicina es la combinación más recomendada para el inmunocomprometido ${ }^{(18,19)}$. No existe consenso oficial acerca de la duración del tratamiento, no obstante, las recomendaciones sugieren que este sea mínimo de tres semanas. Los expertos recomiendan individualizar la terapia: bacteriemia (2 semanas), meningitis (3 semanas), endocarditis ( 4 a 6 semanas) y encefalitis o absceso cerebral (5 a 8 semanas) ${ }^{(18-20)}$.

El estudio presenta las limitaciones propias de todo estudio retrospectivo al basar sus resultados en los registros clínicos. Por ello, no se logró obtener con precisión la mortalidad

\section{REFERENCIAS BIBLIOGRÁFICAS}

1. Mook P, O'Brien SJ, Gillespie IA. Concurrent conditions and human listeriosis, England, 1999-2009. Emerg Infect Dis. 2011;17(1):38-43. doi: 10.3201/eid1701.101174.

2. Limmahakhun S, Chayakulkeeree M. Listeria monocytogenes brain abscess: two cases and review of the literature. Southeast Asian J Trop Med Public Health. 2013;44(3):468-78.

3. Ramsakal A, Nadiminti H, Field T, Vincent AL, Greene JN, Lall Dass $\mathrm{V}$, et al. Listeria infections in cancer patients. Infect Med 2004;21(7):345-9.

4. Anaissie E, Kontoyiannis DP, Kantarjian H, Elting L, Robertson LE, Keating M. Listeriosis in patients with chronic lymphocytic leukemia who were treated with fludarabine and prednisone. Ann Intern Med. 1992;117(6):466-9. doi: 10.7326/0003-4819-117-6-466.

5. Goulet V, Hebert M, Hedberg C, Laurent E, Vaillant V, De Valk H, Desenclos JC. Incidence of listeriosis and related mortality among groups at risk of acquiring listeriosis. Clin Infect Dis. 2012;54(5):65260. doi: $10.1093 / \mathrm{cid} / \mathrm{cir} 902$.

6. Scallan E, Hoekstra RM, Angulo FJ, Tauxe RV, Widdowson MA, Roy SL, Jones JL, Griffin PM. Foodborne illness acquired in the United States--major pathogens. Emerg Infect Dis. 2011;17(1):7-15. doi: 10.3201/eid1701.p11101.

7. Vaillant V, de Valk H, Baron E, Ancelle T, Colin P, Delmas MC, Dufour B, Pouillot R, Le Strat Y, Weinbreck P, Jougla E, Desenclos JC. Foodborne infections in France. Foodborne Pathog Dis. 2005;2(3):221-32. doi: 10.1089/fpd.2005.2.221.

8. Espinoza A, De La Torre M, Salinas M, Sánchez V. Determinación de Listeria monocytogenes en quesos frescos de producción artesanal que se expenden en los mercados del distrito de Ica, enero - marzo 2003. Rev Peru Med Exp Salud Publica. 2004;21(2):71-5.

9. Siegman-Igra Y, Levin R, Weinberger M, Golan Y, Schwartz D, Samra $Z$, et al. Listeria monocytogenes infection in Israel and review of cases worldwide. Emerg Infect Dis. 2002;8(3):305-10. doi: 10.3201/ eid0803.010195.

10. Cairns BJ, Payne RJ. Sudden increases in listeriosis rates in England and Wales, 2001 and 2003. Emerg Infect Dis. 2009;15(3):465-8. doi: 10.3201/eid1503.071432. atribuible a listeriosis. Además, a raíz de limitaciones técnicas temporales en el departamento de microbiología, no se logró la identificación de la especie de listeria en todos los aislamientos.

En conclusión, el presente estudio muestra que la listeriosis tiene un comportamiento agresivo y diseminado en los pacientes oncológicos, conllevando elevada morbimortalidad, por lo que debe sospecharse este diagnóstico preferentemente en todo paciente con neoplasia hematológica que acuda con sepsis y compromiso neurológico de instalación aguda.

Contribuciones de autoría: AC, JV, AH, DP y LC han participado en la concepción del artículo y su redacción. AC realizó la recolección y resumen de los datos. AC, JV, AH, DP, LC y WV realizaron el análisis en interpretación de los resultados. AH, JV, DP, WV y LC hicieron la revisión crítica del artículo. AC, JV, AH y DP realizaron las correcciones del artículo. Todos los autores aprobaron la versión final del manuscrito y asumen responsabilidad frente a todos los contenidos del manuscrito.

Financiamiento: Autofinanciado.

Conflictos de interés: Los autores declaran no tener conflictos de interés.

11. Sedano R, Fica A, Guiñez D, Braun S, Porte L, Dabanch J, et al. Infections by Listeria monocytogenes. Rev Chilena Infectol. 2013;30(4):41725. doi: 10.4067/S0716-10182013000400011.

12. Safdar A, Armstrong D. Listeriosis in patients at a comprehensive cancer center, 1955-1997. Clin Infect Dis. 2003;37(3):359-64. doi: $10.1086 / 376631$.

13. Rivero GA, Torres HA, Rolston KV, Kontoyiannis DP. Listeria monocytogenes infection in patients with cancer. Diagn Microbiol Infect Dis. 2003;47(2):393-8. doi: 10.1016/s0732-8893(03)00116-0.

14. Barocci S, Mancini A, Canovari B, Petrelli E, Sbriscia-Fioretti E, Licci A, et al. Listeria monocytogenes meningitis in an immunocompromised patient. New Microbiol. 2015;38(1):113-8.

15. Vázquez-Boland JA, Kuhn M, BercheP, Chakraborty T, Domínguez-Bernal $\mathrm{G}$, Goebel W, et al. Listeria pathogenesis and molecular virulence determinants. Clin Microbiol Rev. 2001;14(3):584-640. doi: 10.1128/CMR.14.3.584640.2001.

16. Arias Miranda IM, Nuño Mateo FJ, Noval Menéndez J, Fonseca Aizpuru EM, Menéndez Calderón MJ. Listeriosis en el adulto. Revisión de 10 casos. An Med Interna. 2004;21:75-78.

17. Guillet C, Join-Lambert O, Le Monnier A, Leclercq A, Mechai F, Mamzer-Bruneel MF, et al. Human listeriosis caused by Listeria ivanovii. Emerg Infect Dis. 2010;16(1):136-8. doi: 10.3201/ eid1601.091155.

18. Mitjà O, Pigrau C, Ruiz I, Vidal X, Almirante B, Planes AM, et al. Predictors of mortality and impact of aminoglycosides on outcome in listeriosis in a retrospective cohort study. J Antimicrob Chemother. 2009;64(2):416-23. doi: 10.1093/jac/dkp180.

19. Lorber B. Listeria monocytogenes. In: Principles and Practice of Infectious Diseases, 7th ed, Mandell GL, Bennett JE, Dolin R (Eds), Churchill Livingstone, Philadelphia 2010. p.2707.

20 Bartt R. Listeria and atypical presentations of Listeria in the central nervous system. Semin Neurol. 2000;20(3):361-73. doi: 10.1055/s2000-9398. 